\title{
Smart Classroom utilizando dispositivos IoT: uma revisão sistemática da literatura
}

\author{
Vinícius Lopes $^{1}$, Roseclea D. Medina ${ }^{1}$, Giliane Bernardi ${ }^{1}$, Felipe B. Nunes $^{2}$ \\ ${ }^{1}$ Universidade Federal de Santa Maria (UFSM) - Av. Roraima no 1000 \\ Caixa Postal 5082 - CEP 97105-900 - Santa Maria - RS - Brasil \\ ${ }^{2}$ Antonio Meneghetti Faculdade (AMF) \\ Est. Recanto Maestro no 338 - CEP 97200-000 - Restinga Sêca - RS - Brasil \\ \{vlopes, rose, giliane\}@inf.ufsm.br, nunesfb@gmail.com
}

\begin{abstract}
The teaching and learning process in classrooms has been getting new frontiers with the introduction of a Smart Classroom concept: intelligent and context-aware classrooms that promote teaching and learning in a convenient and ubiquitous way. Allied with that, IoT devices have a great variety of ideas for use in Smart Classrooms, where several applications of systems have been contributing to the development of new Smart Classroom technologies. This paper outlines the results of a systematic literature review where different systems that use these technologies are identified, and in that study, different paradigms, perspectives, and approaches on the subject are recognized.
\end{abstract}

Resumo. O processo de ensino-aprendizagem em salas de aula vem ganhando novas fronteiras com a introdução do conceito de Smart Classroom: salas de aulas inteligentes e conscientes de contexto, que promovem a aprendizagem de forma ubíqua. Aliado a isso, o uso de dispositivos IoT possui uma grande variedade de propostas no contexto de Smart Classrooms, onde diversas aplicações de sistemas com novas tecnologias vem sendo desenvolvidos nos últimos anos. Este artigo apresenta o resultado de uma Revisão Sistemática de Literatura onde são identificados diferentes sistemas que aplicam essas tecnologias e por meio desse estudo são reconhecidos paradigmas, perspectivas e abordagens referentes ao tema.

\section{Introdução}

Países em desenvolvimento vêm utilizando Tecnologias de Informação e Comunicação como forma de aumentar a qualidade da educação em sala de aula, podendo ser usadas para diversificar o processo de aprendizagem, pesquisa e gerenciamento de alunos. Uma dessas tecnologias é a Internet, que vem sendo utilizada para conectar dispositivos implementados em salas de aula com dispositivos integrados no cotidiano dos professores e alunos [Sari et al. 2017].

Estes dispositivos representam o paradigma de Internet das Coisas (ou IoT, sigla definida pela expressão do termo original Internet of Things). O paradigma de IoT é definido por [Chebudie et al. 2014], como um conjunto de dispositivos unicamente endereçáveis interconectados através da Internet, podendo possuir sensores que capturam dados contínuos e discretos do mundo real a fim de cumprir uma grande variedade de funções. 
VII Congresso Brasileiro de Informática na Educação (CBIE 2018)

Anais do XXIX Simpósio Brasileiro de Informática na Educação (SBIE 2018)

Tais dispositivos promovem o aprimoramento do ensino-aprendizagem em salas de aula, aproximando-as ainda mais de uma Smart Classroom, conceito que é definido, por [Liu et al. 2017], como uma sala de aula inteligente onde há consciência de contexto e gerência de seu ambiente de aprendizagem, e que, a partir disso, apresenta conteúdo educacional de forma eficiente, que torna conveniente o acesso a recursos de aprendizado e que promove interação entre os alunos.

Considerando o crescimento da utilização de dispositivos IoT em diferentes contextos do cotidiano, como em casa, na rua, no ambiente de trabalho ou no campus, surge a oportunidade de adotar tais dispositivos com o propósito de tornar as salas de aula cada vez mais inteligentes, ubíquas e próximas da definição de uma Smart Classroom. Justifica-se, então, a necessidade de uma pesquisa mais detalhada sobre o tema, a fim de mapear de que forma estes dispositivos já vêm contribuindo para o ensino e a aprendizagem em Smart Classrooms.

Busca-se, com esta pesquisa, fazer um levantamento de sistemas existentes que se utilizam de dispositivos IoT em Smart Classrooms, quais tecnologias e inovações esses sistemas possuem de forma a contribuir para o ensino e aprendizagem, assim como também descobrir de que forma esses sistemas vêm sendo avaliados. Tal pesquisa foi realizada em forma de revisão sistemática da literatura, processo cujas definições serão explanadas na seção de metodologia.

\section{Trabalhos Relacionados}

Ao longo da pesquisa, foram encontrados outros trabalhos que também consistem em estudos secundários e que se relacionam com o tema da pesquisa atual. Suas contribuições foram estudadas e analisadas a fim de auxiliar nas considerações e conclusões deste estudo.

[Muhamad et al. 2017] apresentam uma revisão sistemática de literatura que define o conceito de Smart Campus, identifica suas tecnologias e áreas de aplicação, assim como modelos padronizados de projetos e aplicações já desenvolvidas. A pesquisa encontrou aplicações nos mais diferentes domínios, categorizando-os em seis: iLearning, iSocial, iManagement, iGovernance, iHealth e iGreen, sendo o domínio de iLearning aquele onde mais houveram aplicações. Concluiu-se que, no contexto de um campus inteligente, as maiores tendências estão relacionadas ao desenvolvimento de soluções para o ensino e a aprendizagem, bem como assessoramento de estudantes, gerenciamento de estilos de aprendizagem e ensino personalizado.

Já em [Henrique and Araujo 2018], é apresentado um mapeamento sistemático de literatura, com propostas de soluções para campus inteligentes, discutindo metodologias, práticas e tecnologias aplicáveis neste contexto. Por meio deste mapeamento, foi possível classificar tecnologias em um campus inteligente considerando quatro eixos: Serviços, Ensino e Aprendizagem, Governança e Gestão e Infraestrutura. Tratando-se exclusivamente de ensino e aprendizagem, foi constatado, ao final da pesquisa, que Tecnologias de Informação e Comunicação (TIC) podem enriquecer os ambientes educacionais. Foi ressaltada, também, a importância do incentivo à interação social, compartilhamento de conteúdo e aprendizagem colaborativa.

Os trabalhos citados tratam da aplicação de TIC em Smart Campus em diferentes contextos. Diferente destes, esta pesquisa aprofunda-se no contexto de Smart Classrooms, 
VII Congresso Brasileiro de Informática na Educação (CBIE 2018)

Anais do XXIX Simpósio Brasileiro de Informática na Educação (SBIE 2018)

com foco em ensino e aprendizagem, fazendo uma revisão sistemática da literatura com a finalidade de identificar sistemas que utilizem dispositivos IoT para o desenvolvimento de tecnologias neste contexto.

\section{Metodologia}

Uma revisão sistemática da literatura consiste em um processo de identificação, avaliação e interpretação de toda a pesquisa feita sobre determinado assunto ou fenômeno. [Kitchenham 2004] estabeleceu, em seu trabalho, padrões, diretrizes e processos para que uma revisão da literatura pudesse ser considerada abrangente, relevante e imparcial. Inicialmente desenvolvida para pesquisa na área da medicina, foi adaptada por [Budgen and Brereton 2006], para pesquisa na área da engenharia e desenvolvimento de software.

Com base nestas características, decidiu-se aplicar este método de pesquisa para encontrar pesquisas relacionadas ao tema proposto. Em um primeiro momento foram definidos elementos como as questões de pesquisa, palavras-chave, string de busca, fontes de publicações acadêmicas e critério de inclusão e exclusão de estudos. Este processo é detalhado na subseção 3.1.

Em seguida, foram estruturadas três etapas de pesquisa. Na primeira etapa, foi realizada a busca inicial por artigos e publicações usando as definições iniciais de pesquisa estabelecidas. Na segunda etapa, houve uma filtragem primária por meio da leitura do título, resumo e palavras-chave dos artigos, a fim de determinar sua relevância com o tema. Em seguida, na terceira etapa, foi feita uma segunda filtragem por meio da leitura completa desses artigos, com o propósito de determinar se os mesmos contém todas as informações necessárias para os fins quantitativos e qualitativos desta pesquisa. Em todas as etapas foram aplicados os critérios de inclusão e exclusão estabelecidos. Este processo é detalhado na subseção 3.2.

\subsection{Definição da Pesquisa}

Como processo inicial, foram estabelecidos os questionamentos a serem respondidos com esta pesquisa. Definiram-se, então, três questões de pesquisa, apresentadas na Tabela 1.

Tabela 1. Questões de pesquisa

\begin{tabular}{|c|c|}
\hline Questão & Descrição \\
\hline QP1 & $\begin{array}{c}\text { Como os sistemas para Smart Classroom, interconectados com } \\
\text { dispositivos IoT, têm sido desenvolvidos? }\end{array}$ \\
\hline QP2 & $\begin{array}{c}\text { Quais funcionalidades, no contexto de Smart Classroom, esses } \\
\text { sistemas possuem ao se integrar com dispositivos IoT? }\end{array}$ \\
\hline QP3 & $\begin{array}{c}\text { Como os sistemas para Smart Classroom, interconectados com } \\
\text { dispositivos IoT, têm sido testados e avaliados? }\end{array}$ \\
\hline
\end{tabular}

Em seguida, como mostra a Tabela 2, foram definidas as palavras-chave que compõem o escopo geral da pesquisa, a fim de definir os próximos passos. Foram usados termos e sinônimos em inglês e português. Com base nas palavras-chave definidas foi criada, então, uma string de busca, a fim de filtrar as pesquisas a serem realizadas. 
VII Congresso Brasileiro de Informática na Educação (CBIE 2018)

Anais do XXIX Simpósio Brasileiro de Informática na Educação (SBIE 2018)

Tabela 2. Palavras-chave e sinônimos

\begin{tabular}{|c|c|}
\hline Palavras-chave & Sinônimos \\
\hline Internet of Things & $\begin{array}{c}\text { Internet das Coisas, IoT, Internet of Everything, IoE, } \\
\text { Internet de Tudo }\end{array}$ \\
\hline Smart Classroom & $\begin{array}{c}\text { Sala de Aula Inteligente, Classroom, Intelligent Classroom, } \\
\text { Smart Campus, Sala de Aula, Campus Inteligente }\end{array}$ \\
\hline
\end{tabular}

A string foi definida como: (("Internet of Things"OR "IoT"OR "Internet das coisas"OR "Internet of Everything"OR "IoE"OR "Internet de tudo") AND ("classroom"OR "smart classroom"OR "intelligent classroom"OR "smart campus"OR "sala de aula"OR "sala de aula inteligente"OR "campus inteligente")).

Em seguida, foram selecionadas seis fontes de pesquisa de conteúdo acadêmico, relacionadas diretamente com as áreas tratadas nesta pesquisa. São elas, as plataformas de biblioteca digital do IEEE Xplore, Scopus, ACM e ScienceDirect, assim como os anais de eventos do CBIE (Congresso Brasileiro de Informática na Educação), SBCUP (Simpósio Brasileiro de Computação Ubíqua e Pervasiva) e SCIRP (Scientific Research Publishing). Tais anais foram escolhidos por abordarem, de forma específica, tópicos de Internet of Things como promotores de ensino-aprendizagem. Para que os resultados obtidos pudessem ser melhor filtrados e interpretados, foram estabelecidos critérios de inclusão e exclusão, representados na Tabela 3.

Tabela 3. Critérios de inclusão e exclusão

\begin{tabular}{|c|c|}
\hline Critérios de Inclusão & Critérios de Exclusão \\
\hline $\begin{array}{c}\text { O estudo analisado apresenta resultados } \\
\text { que correspondem ao objetivo definido } \\
\text { para a pesquisa, com propostas ou } \\
\text { aplicações de sistemas para Smart } \\
\text { Classroom com dispositivos IoT. }\end{array}$ & $\begin{array}{c}\text { O estudo é um relatório técnico, ou } \\
\text { documento no formato de resumo, ou } \\
\text { apresentação, ou chamada de artigo, ou } \\
\text { sumário de conferência, ou estudo } \\
\text { secundário ou terciário. }\end{array}$ \\
\hline $\begin{array}{c}\text { O sistema desenvolvido para a Smart } \\
\text { Classroom apresenta integração com } \\
\text { dispositivos IoT }\end{array}$ & $\begin{array}{c}\text { O estudo não está disponível para uma } \\
\text { leitura completa por ser pago ou de } \\
\text { cunho confidencial. }\end{array}$ \\
\hline O estudo foi publicado entre 2012 e & $\begin{array}{c}\text { Palavras-chave não estão presentes na } \\
\text { estrutura do artigo como um todo. }\end{array}$ \\
\hline 2017. & $\begin{array}{c}\text { O estudo foi escrito e publicado em } \\
\text { língua diferente do inglês e português. }\end{array}$ \\
\hline
\end{tabular}

Com estas diretrizes estabelecidas, iniciou-se, então, o processo de revisão sistemática da literatura, detalhado na subseção a seguir.

\subsection{Condução da Revisão Sistemática}

Inicialmente, na primeira etapa, foi utilizada a string de busca nas plataformas de biblioteca digital do IEEE, ScienceDirect, Scopus e ACM, fazendo as adaptações e filtros necessários a fim de atender os critérios de inclusão e exclusão estabelecidos. Em seguida foi realizada uma busca manual nos anais do CBIE, SCIRP e SBCUP, compreendendo os anos de 2012 a 2017, procurando por publicações que possuíssem como tema as palavraschave da pesquisa. Ao final deste passo, totalizando as buscas em bibliotecas digitais com 
VII Congresso Brasileiro de Informática na Educação (CBIE 2018)

Anais do XXIX Simpósio Brasileiro de Informática na Educação (SBIE 2018)

as em anais de eventos, foram encontradas 209 publicações, que seguiram para as etapas de seleção e filtragem.

Após essa busca, iniciou-se, na segunda etapa, a seleção de publicações, com a leitura dos títulos, resumos e palavras-chave de cada publicação, buscando definir sua a relevância com o tema. Após aplicados novamente os critérios de inclusão e exclusão, restaram 46 publicações que seguiram para a segunda etapa. Ao final desta etapa, os anais do SCIRP e a biblioteca digital ScienceDirect não apresentaram nenhum artigo com os critérios estabelecidos e foram excluídos do processo.

$\mathrm{Na}$ terceira etapa, foi feita a leitura completa das 46 publicações remanescentes. Todas as publicações que passaram para esta etapa se relacionam com as palavras-chave de alguma forma, porém, para fins de seleção, as mesmas foram divididas e classificadas de acordo com três fatores: tipo de pesquisa (se é uma proposta de uma tecnologia, aplicação da mesma ou se é um artigo puramente conceitual), avaliação (se a aplicação ou proposta foi testada e validada de alguma forma) e, por fim, se a publicação apresenta tecnologias para Smart Classroom relacionadas ao ensino e aprendizagem, ou tecnologias mais abrangentes ao conceito de Smart Campus, como otimização de energia, mobilidade, mapeamento de salas de aula e controle de área verde, por exemplo.

Foram, então, eliminadas as publicações que não abrangeram os critérios de inclusão e exclusão estabelecidos, em especial aquelas que apresentavam apenas conceitos, paradigmas e referencial teórico sem nenhuma proposta ou aplicação. Em seguida, foram eliminadas as publicações que apresentavam tecnologias que não relacionavam Smart Classroom focada em processos de ensino e aprendizagem. Após essa filtragem, foram selecionadas 20 publicações para a extração de dados e discussão dos resultados. Para fins quantitativos, a Tabela 4 representa o número de publicações analisadas em cada etapa, para cada fonte de pesquisa.

Tabela 4. Publicações por etapa de pesquisa

\begin{tabular}{|c|c|c|c|}
\hline Fonte de Pesquisa & $\begin{array}{c}\text { Primeira } \\
\text { etapa }\end{array}$ & $\begin{array}{c}\text { Segunda } \\
\text { etapa }\end{array}$ & $\begin{array}{c}\text { Terceira } \\
\text { etapa }\end{array}$ \\
\hline IEEE & 65 & 18 & 6 \\
\hline Scopus & 103 & 16 & 9 \\
\hline Science Direct & 2 & 0 & 0 \\
\hline ACM DL & 19 & 2 & 1 \\
\hline CBIE & 14 & 7 & 3 \\
\hline SBCUP & 5 & 2 & 1 \\
\hline SCIRP & 1 & 0 & 0 \\
\hline Totais & $\mathbf{2 0 9}$ & $\mathbf{4 6}$ & $\mathbf{2 0}$ \\
\hline
\end{tabular}

Todos os artigos referentes às duas últimas etapas do processo, assim como detalhes da classificação dos mesmos, podem ser visualizados em uma planilha pelo link: https://goo.gl/y3yDQU. Os resultados dessa pesquisa serão apresentados, classificados e discutidos na seção a seguir. 
VII Congresso Brasileiro de Informática na Educação (CBIE 2018)

Anais do XXIX Simpósio Brasileiro de Informática na Educação (SBIE 2018)

\section{Discussão dos Resultados}

Ao final desta pesquisa, foram encontrados 20 artigos relevantes ao tema, de 2012 a 2017. Foi encontrada uma quantidade relativamente baixa de artigos e $80 \%$ dos mesmos foram publicados nos últimos dois anos da pesquisa. Logo, constatou-se que Smart Classroom integrada com dispositivos IoT é um tema emergente na área acadêmica e que vem obtendo uma rápida ascensão em publicações.

Em relação à primeira questão de pesquisa, percebeu-se que, dos 20 artigos selecionados, 6 apresentam propostas de novas tecnologias em sala de aula pelo desenvolvimento de arquiteturas para sistemas, aplicações e ferramentas de hardware, como pode ser visto, respectivamente, em [Silva et al. 2016], [Chan et al. 2017] e [Merino et al. 2016]. Por outro lado, os outros 14 artigos apresentam a aplicação de tecnologias por meio do desenvolvimento de uma plataforma, um framework ou um software, como os vistos em [Iqbal 2016], [Albiero et al. 2017] e [Szedmina et al. 2015], respectivamente. Conclui-se que as tecnologias para sala de aula usando dispositivos IoT estão sendo implementadas e testadas de acordo com o desenvolvimento dos recursos tecnológicos.

Deve-se ressaltar, contudo, a necessidade de padronização e reutilização dos sistemas que implementam essas tecnologias, uma vez que nenhum dos sistemas analisados consideraram a existência uns dos outros durante seu desenvolvimento, ato que proporciona diferentes soluções para um mesmo problema e que gera retrabalho, muitas vezes desnecessário. Em [Fuzeto and Braga 2017] é proposta a UL-SOPL, uma linha de produtos orientada a serviços visando a facilidade de implementação de sistemas de aprendizagem ubíquos utilizando dispositivos IoT. Um projeto dessa natureza pode ser visto como uma possível solução para esta questão de padronização de sistemas, visto que tal padronização pode vir a promover o reúso de tecnologias já existentes e aumentar o escopo de desenvolvimento das mesmas, proporcionando tecnologias cada vez mais refinadas e avançadas.

Referente à segunda questão de pesquisa, foram estabelecidas quatro abordagens diferentes no âmbito de ensino e aprendizagem em Smart Classroom. São elas: técnicas e estilos de aprendizagem, inclusão e acessibilidade, controle de frequência, e atenção, foco e participação em sala de aula. Tais abordagens são baseadas na classificação em diferentes eixos de tecnologias para Smart Campus definida por [Henrique and Araujo 2018]. A Figura 1 ilustra a categorização das 20 publicações referente a essas abordagens. Tal categorização teve como propósito quantificar os tipos de sistemas desenvolvidos a fim de permitir uma pesquisa exploratória, descritiva e, consequentemente, explicativa sobre os mesmos.

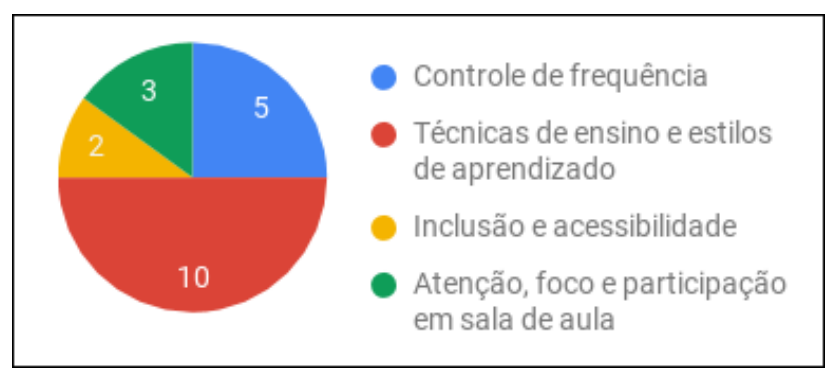

Figura 1. Abordagens em Smart Classroom 
VII Congresso Brasileiro de Informática na Educação (CBIE 2018)

Anais do XXIX Simpósio Brasileiro de Informática na Educação (SBIE 2018)

Para uma Smart Classroom, controle de frequência refere-se a automatização da frequência de um aluno em sala de aula, seja pela presença em ambiente físico, ou interação em um ambiente virtual em determinados períodos de tempo [Chan et al. 2017]. Pode-se citar, como exemplo, o OnBee de [Chamon et al. 2014], um sistema ubíquo capaz de realizar o registro automático da presença de alunos em sala de aula não apenas em sua entrada e saída, mas durante todo o período de aula. Foram realizados experimentos em um ambiente simulado de uma sala de aula, referentes ao ciclo de trabalho desse sistema e a durabilidade de seus componentes. Tais experimentos comprovaram sua funcionalidade, assim como sua viabilidade técnica e financeira.

A abordagem de atenção, foco e participação em sala de aula, no contexto de Smart Classroom, pode ser definida como a utilização de sensores para medir e avaliar fatores referentes ao rendimento do aluno em sala de aula, a fim de garantir um aprendizado mais eficiente [Iqbal 2016]. Neste contexto, analisando os trabalhos, podemos citar [Ghazal et al. 2017], onde foi desenvolvido um framework para analisar o nível de participação de pessoas em um encontro por meio de captadores de áudio. Uma finalidade proposta para este sistema é a de quantificar a participação do aluno em uma sala de aula, com o intuito de elaborar iniciativas e soluções para aumentá-la. O framework foi testado em diferentes ambientes controlados, onde foi concluído que sua utilização promove, em média, um aumento de $15 \%$ na acurácia da quantificação de participação dos indivíduos.

Inclusão e acessibilidade em Smart Classroom é uma abordagem referente à inclusão de pessoas com deficiência em uma sala de aula, com a utilização de dispositivos e sensores adaptáveis para cada aluno, levando em conta possíveis deficiências ao fornecer acesso e ao apresentar conteúdo, a fim de assegurar igualdade de ensino para todos os alunos [Shi et al. 2017]. Nesta abordagem, [Silva et al. 2016] propuseram um sistema computacional utilizando dispositivos IoT e computação vestível com o intuito de proporcionar a inclusão social de surdos em sala de aula através do ensino-aprendizagem de libras. Destaca-se também o trabalho de [Shi et al. 2017], onde foi desenvolvido um sistema, também utilizando dispositivos IoT e computação vestível, para monitorar interações entre crianças com autismo em uma pré-escola a fim de identificar anomalias de comportamento e, consequentemente, realizar intervenções de pais ou professores, caso necessário.

Técnicas de ensino e estilos de aprendizado em Smart Classroom correspondem a uma abordagem em que se busca automatizar soluções de adaptação de ensino ao aluno, baseando-se na identificação de seu estilo de aprendizagem [Shapsough and Zualkernan 2017]. Como exemplo desta abordagem, ressalta-se o trabalho de [Cheng and Liao 2012], aonde foi desenvolvido o sistema ELLLA, que combinou técnicas de aprendizado analítico com o uso de dispositivos IoT para conduzir uma análise do processo de aprendizado de estudantes a fim de estabelecer um ambiente de ensino eficaz.

Com base nos dados levantados referentes às diferentes abordagens em Smart Classroom, pode-se constatar que tecnologias de ensino e estilos de aprendizagem caracterizam a maior parte dos sistemas que vêm sendo desenvolvidos, representando 50\% das abordagens encontradas nas publicações. Embora este seja o foco no cenário atual, deve ser ressaltada a necessidade de investimento em outras abordagens, em especial a de inclusão e acessibilidade, que, embora represente a menor parcela de sistemas encontrados, é de suma importância em busca de um cenário educacional inclusivo e globalizado. 
VII Congresso Brasileiro de Informática na Educação (CBIE 2018)

Anais do XXIX Simpósio Brasileiro de Informática na Educação (SBIE 2018)

Na Tabela 5 são listadas todas as 20 publicações encontradas por esta pesquisa, categorizadas por suas respectivas abordagens no contexto de Smart Classroom.

Tabela 5. Publicações encontradas ao final do processo

\begin{tabular}{|c|c|}
\hline $\begin{array}{c}\text { Abordagens para Smart } \\
\text { Classroom }\end{array}$ & Publicações \\
\hline $\begin{array}{c}\text { Atenção, foco e } \\
\text { participação em sala de aula }\end{array}$ & [Lim et al. 2017]; [Iqbal 2016]; [Ghazal et al. 2017] \\
\hline Controle de frequência & $\begin{array}{c}\text { [G. et al. 2016]; [Albiero et al. 2017]; [Atabekov 2016] } \\
\text { [Chan et al. 2017]; [Chamon et al. 2014] }\end{array}$ \\
\hline Inclusão e acessibilidade & [Shi et al. 2017]; [Silva et al. 2016] \\
\hline $\begin{array}{c}\text { Técnicas de ensino e estilos } \\
\text { de aprendizado }\end{array}$ & $\begin{array}{c}\text { [Shapsough and Zualkernan 2017]; } \\
\text { [Merino et al. 2016]; [Palma et al. 2014] } \\
\text { [Cheng and Liao 2012]; [Sorrentino et al. 2016]; } \\
\text { [Veeramanickam and Mohanapriya 2017] } \\
\text { [Zhamanov et al. 2017]; [Fuzeto and Braga 2017] }\end{array}$ \\
\hline
\end{tabular}

Por fim, respondendo a questão de pesquisa 3, verificou-se que dentre os sistemas desenvolvidos, os testes e validações foram realizados de diferentes formas. Em 7 (sete) publicações foi observada a validação por meio da coleta e análise de dados em salas de aula, em outras 5 (cinco) publicações a avaliação e a validação foram feitas testando a eficiência dos sistemas em simulações e ambientes controlados. Por fim, 5 (cinco) publicações relataram o uso de questionários de avaliação com usuários para realizar a validação de seu sistema. O que percebe-se com isto é que há uma discrepância na forma como essas tecnologias são testadas e avaliadas, não havendo nenhum instrumento de coleta de dados e avaliação específico para cenários de ensino-aprendizagem.

\section{Considerações Finais}

O uso de dispositivos IoT integrados a diferentes aspectos do cotidiano, seja para lazer, trabalho ou estudo tem obtido um rápido crescimento nos últimos anos. No contexto de Smart Classrooms, novas tecnologias usando esses dispositivos também vem obtendo um crescimento considerável. Com isso, percebeu-se a necessidade de fazer um estudo mais aprofundado sobre o assunto a fim de compreender como essas novas tecnologias têm sido desenvolvidas e avaliadas. Para tal estudo, foi realizada uma revisão sistemática da literatura, por meio da qual foram encontradas e analisadas 20 publicações acerca do tema.

Respondidas as questões de pesquisa propostas, constatou-se que a implementação de dispositivos IoT em Smart Classrooms é um tema emergente na academia, com uma grande variedade de propostas e aplicações de sistemas sendo desenvolvidos nos últimos anos. Todavia, percebe-se uma dispersão nos métodos de planejamento, desenvolvimento e avaliação desses sistemas. Com isso, surge a necessidade de padronizar e adaptar as soluções tecnológicas já desenvolvidas a fim de evitar retrabalho em soluções para problemas já resolvidos, assim como também para garantir a qualidade dos processos de validação efetuados nesses sistemas. 
VII Congresso Brasileiro de Informática na Educação (CBIE 2018)

Anais do XXIX Simpósio Brasileiro de Informática na Educação (SBIE 2018)

Constatou-se, também, que o foco tem sido maior em técnicas de ensino e estilos de aprendizagem nos sistemas analisados e que ainda há muito a ser desenvolvido na questão de inclusão, acessibilidade e monitoramento de salas de aula. Por fim, como trabalho futuro, propõe-se um novo levantamento de dados a fim de estabelecer padrões e diretrizes tanto para o desenvolvimento quanto para a avaliação de tecnologias para salas de aula inteligentes.

\section{Referências}

Albiero, F., Lima, J. C., and Albiero, F. (2017). Bluetapp um aplicativo móvel para registro da frequência acadêmica através da tecnologia bluetooth. In Anais dos Workshops do Congresso Brasileiro de Informática na Educação, volume 6, page 1289.

Atabekov, A. (2016). Internet of things-based smart classroom environment. In Proceedings of the 31st Annual ACM Symposium on Applied Computing, pages 746-747.

Budgen, D. and Brereton, P. (2006). Performing systematic literature reviews in software engineering. In Proceedings of the 28th International Conference on Software Engineering, ICSE '06, pages 1051-1052, New York, NY, USA. ACM.

Chamon, J. P. M., Souza, T. D. V., Rosa, A. S., and Mini, R. A. F. (2014). Registro ubíquo de controle acadêêmico: Localizacão em ambiente interno utilizando ciclo de trabalho dinâmico. In Simpósio Brasileiro de Computação Ubíqua e Pervasiva.

Chan, E., Othman, M., and Razak, M. A. (2017). Iot based smart classroom system. Journal of Telecommunication, Electronic and Computer Engineering (JTEC), 9(39):95-101.

Chebudie, A. B., Minerva, R., and Rotondi, D. (2014). Towards a definition of the internet of things (iot).

Cheng, H.-C. and Liao, W.-W. (2012). Establishing an lifelong learning environment using iot and learning analytics. In Advanced Communication Technology (ICACT), 2012 14th International Conference on, pages 1178-1183. IEEE.

Fuzeto, R. and Braga, R. (2017). Proposta de uma linha de produtos de software para sistemas de aprendizagem ubíquos com internet das coisas. In Anais dos Workshops do Congresso Brasileiro de Informática na Educação, volume 6, page 1232.

G., M., R., J. K., and Bijlani, K. (2016). A smart phone integrated smart classroom. In 2016 10th International Conference on Next Generation Mobile Applications, Security and Technologies (NGMAST), pages 88-93.

Ghazal, M., Khalil, A., Al Hadithy, T., Al Tarabsheh, A., and Ali, S. (2017). Intellimeet: Collaborative mobile framework for automated participation assessment. In Future Internet of Things and Cloud Workshops (FiCloudW), 2017 5th International Conference on, pages 227-232. IEEE.

Henrique, F. and Araujo, R. (2018). Campus inteligentes: Conceitos, aplicações, tecnologias e desafios.

Iqbal, M. Z. (2016). Enhancing classroom engagement through smart phone based paper marking solution. In Proceedings of the 6th International Conference on the Internet of Things, pages 161-162. ACM. 
VII Congresso Brasileiro de Informática na Educação (CBIE 2018)

Anais do XXIX Simpósio Brasileiro de Informática na Educação (SBIE 2018)

Kitchenham, B. (2004). Procedures for performing systematic reviews. Keele, UK, Keele University, 33(TR/SE-0401):28.

Lim, J. H., Teh, E. Y., Geh, M. H., and Lim, C. H. (2017). Automated classroom monitoring with connected visioning system. In Asia-Pacific Signal and Information Processing Association Annual Summit and Conference (APSIPA ASC), 2017, pages 386-393.

Liu, D., Huang, R., and Wosinski, M. (2017). Smart Learning in Smart Cities.

Merino, P. P., Ruiz, E. S., Fernandez, G. C., and Gil, M. C. (2016). A wireless robotic educational platform approach. In 2016 13th International Conference on Remote Engineering and Virtual Instrumentation (REV), pages 145-152.

Muhamad, W., Budi, K. N., Suhardi, S., and Yazid, S. (2017). Smart campus features, technologies, and applications: A systematic literature review. pages 384-391.

Palma, D., Agudo, J. E., Sánchez, H., and Macías, M. M. (2014). An internet of things example: Classrooms access control over near field communication. In Sensors.

Sari, M. W., Wahyu Ciptadi, P., and Hardyanto, R. (2017). Study of smart campus development using internet of things technology. 190:012032.

Shapsough, S. Y. and Zualkernan, I. A. (2017). A voice-based mobile system for generating stallings-type class observations. In 2017 IEEE 17th International Conference on Advanced Learning Technologies (ICALT), pages 457-459.

Shi, Y., Das, S., Douglas, S., and Biswas, S. (2017). An experimental wearable iot for data-driven management of autism. In 2017 9th International Conference on Communication Systems and Networks (COMSNETS), pages 468-471.

Silva, R. A., Lima, L., and Bastos, R. (2016). Aperfeiçoando o aprendizado de libras utilizando elementos de internet das coisas. In Anais dos Workshops do Congresso Brasileiro de Informática na Educação, volume 5, page 1364.

Sorrentino, F., Spano, L. D., and Scateni, R. (2016). Internet of t (eac) hings: Assessing children's learning in the iot era. In CEUR WORKSHOP PROCEEDINGS, volume 1602, pages 31-35. CEUR-WS.

Szedmina, L., Molcer, P. S., Simon, J., and Čović, Z. (2015). Clicking for business english success. In Intelligent Systems and Informatics (SISY), 2015 IEEE 13th International Symposium on, pages 313-317. IEEE.

Veeramanickam, M. and Mohanapriya, M. (2017). Iot enabled futurus smart campus with effective e-learning: i-campus. GSTF Journal of Engineering Technology (JET), 3(4).

Yuqiao, Y. and Kanhua, Y. (2016). Construction of distance education classroom in architecture specialty based on internet of things technology. International Journal of Emerging Technologies in Learning (iJET), 11(05):56-61.

Zhamanov, A., Sakhiyeva, Z., Suliyev, R., and Kaldykulova, Z. (2017). Iot smart campus review and implementation of iot applications into education process of university. In Electronics, Computer and Computation (ICECCO), 2017 13th International Conference on, pages $1-4$. IEEE. 